\title{
Mathematical Model For Human ReSOURCES PlanNing in the Production Process
}

\author{
Aleksandra Kostic, Bogdan Maric, Melisa Kustura \& Valentina Timotic
}
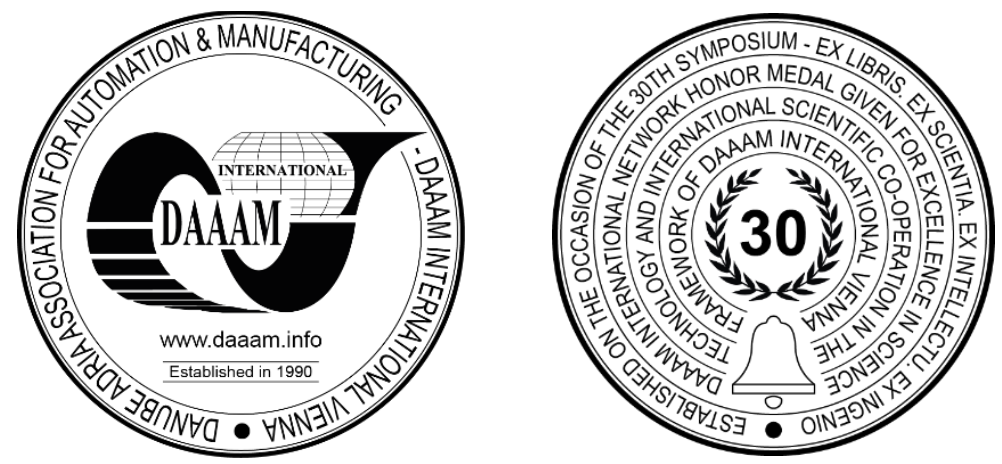

This Publication has to be referred as: Kostic, A[leksandra]; Maric, B[ogdan]; Kustura, M[elisa] \& Timotic, V[alentina] (2021). Mathematical Model for Human Resources Planning in the Production Process, Proceedings of the 32nd DAAAM International Symposium, pp.0004-0009, B. Katalinic (Ed.), Published by DAAAM International, ISBN 978-3-902734-33-4, ISSN 1726-9679, Vienna, Austria

DOI: $10.2507 / 32$ nd.daaam.proceedings.001

\begin{abstract}
Problems of planning, management and optimal use of available human resources in the production process are very important for rational business management. The training of workers to service more jobs or vast number of machines with the aim of reducing the number of workers in the production process, thus balancing the workload, is an economic imperative. With the COVID 19 pandemic, the problem of reducing the number of workers in the production plant became even more notable. This paper presents a mathematical model for human resource planning. The application of the model was examined on the work potential, in terms of the interdisciplinarity of individuals, in the production system.
\end{abstract}

Keywords: Management; planning; human potential; mathematical model.

\section{Introduction}

The world of the new millennium is characterized by globalization, liberalization, technological development and lifelong learning. In such conditions, modern production systems were, until two years ago, consumer-oriented, which were key factors in planning the work process in production systems. Consumers were in a position to set high criteria in terms of product price, quality and time to market, because they had a large selection of products from different manufacturers at their disposal. We should not forget the fact that in the last decade, the world economy has been shaken by the economic crisis and recession, which is especially reflected in production processes and equally affects small and large companies. In the last two years, we have witnessed the COVID 19 pandemic, which significantly affects production processes, which were affected by lockdown, epidemiological measures and the requirement to carry out the production process with as few workers as possible in production halls. In such conditions, great demands are placed on the systems of production process management, especially human resources, with the aim of more rational business. The COVID 19 pandemic has once again put human resources on an almost equal footing with consumers in terms of the orientation of production systems.

During the last years of the last century, one became aware of all the shortcomings of the classical system of production process management and in response to this situation, the Lean concept was developed as a source of improving efficiency and effectiveness in work processes. The development of this approach was initiated by Lean Production. 
This represented a breakthrough in more significant process analyses in order to improve production and the factory environment in which products are created. Lean production has changed the opinion and behaviour of participants in work processes and created an environment in which work is focused on: work process, elimination of losses in the work process, permanent training of employees at all levels and in all functions in order to achieve shorter delivery times and cheaper products. In this way, an ambience was created in the factories, which represents the Lean concept or doctrine in the way of managing and organizing production plants. Many authors state the efficiency in the development of organizational and managerial structures of production or industrial systems called "efficiency of the third dimension" which has a basic role in the Lean concept. The basic characteristics of managers who lead the work process in the Lean concept are dynamic behaviour, quick decision-making and teamwork focused on products, customers and the market, and training and maintaining employees to respond to environmental conditions. More about the Lean concept can be found in [1]. Due to the great advantages provided by the Lean concept, it is still most often used today to solve various problems in production, which can be seen from the works [3], [6], [7], [8].

Workers are seen as an economic factor of the production process and therefore the problem of planning, management and optimal use of available human resources plays an important role in the production economy. To manage work potential, the tool of the Lean concept - Nagara System can be applied, which in human resources imperatively requires greater interdisciplinarity of the individual in the interest of overall efficiency. Experimental research Lean concepts - Nagara System on the distribution and duration of the overhaul cycle in the process of repairing a turbojet engine (TMM), the redistribution of jobs to workers trained for more jobs is shown in [4], [5].

Experiences with the pandemic have again put the worker in an important place in terms of human resource management. Namely, previous experiences show that such emergencies are possible and that the production system must adapt to new challenges such as: reducing the number of workers in one shift, shortening the duration of the shift, enabling social distance between workers and the fact that for each job there must be at least two workers trained to perform the same task. Due to the unpredictability of viral infections as well as their duration, production must not be allowed to depend on a single worker who may be incapacitated by the disease to perform tasks. Therefore, it is necessary to develop a simpler mathematical model, without complex diagrams, which will effectively deploy workers in order to increase production efficiency and overcome production problems in emergency conditions such as a pandemic. This is also the subject of our paper.

The work is organized as follows: Section 2 discusses the position of workers in the new organizational concept of production systems as well as the conditions of the pandemic, the demands of management to the worker and the worker to management. The advantages of lifelong learning as well as the impact of the new organizational concept on employee motivation and effectiveness are also considered. The mathematical model of worker redistribution in terms of achieving higher productivity and compliance with epidemiological measures will be discussed in Section 3 . The proposed mathematical model is characterized by ease of application and clear dynamic, without the use of complex graphs. In section 4, the mathematical model is applied and tested on a specific production (overhaul) process. Section 5 provides a critical consideration of the application of the mathematical model and guidelines for further research.

\section{Human resources}

The quality and quantity of the workforce is changing significantly. The requirements of the employee towards the employer as well as the requirements of the management towards the employee are also changing. Therefore, it is necessary to analyse the demands of workers and management. Worker requirements are usually the following: guaranteed wages, more favourable working conditions, greater responsibility and opportunities for decision-making in the work process, protection of workers at work, permanent training and education and implementation of epidemiological measures in order to reduce COVID 19 infection. On the other hand, the requirements of the employer are: more efficient production, interdisciplinary work potential that is able to perform a variety of activities with the aim of increasing the level of utilization of working time to meet market demands. We will give one simple example where the production bottleneck can be easily overcome by redirecting workers to different working operation. Workers and the duration of the operation are marked in series production with $W_{1}, W_{2}, W_{3}$ and $W_{4}$. The graph shows the sequence of their operations and indicates the time of their execution. First, a situation is shown where each worker is trained to perform only operations that are performed recursively one after the other.

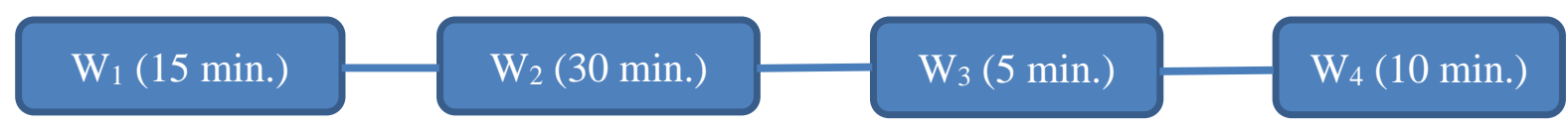

Fig. 1. Diagram 1

It is clear that the bottleneck in production has occurred among $W_{2}$ workers who, due to the length of the operation, reduce the capacity of the entire chain with their limited production capacity.

If now workers $W_{2}$ and $W_{3}$ are both trained for both operations, and if they perform a fusion of their activities we get the following diagram 2 . 


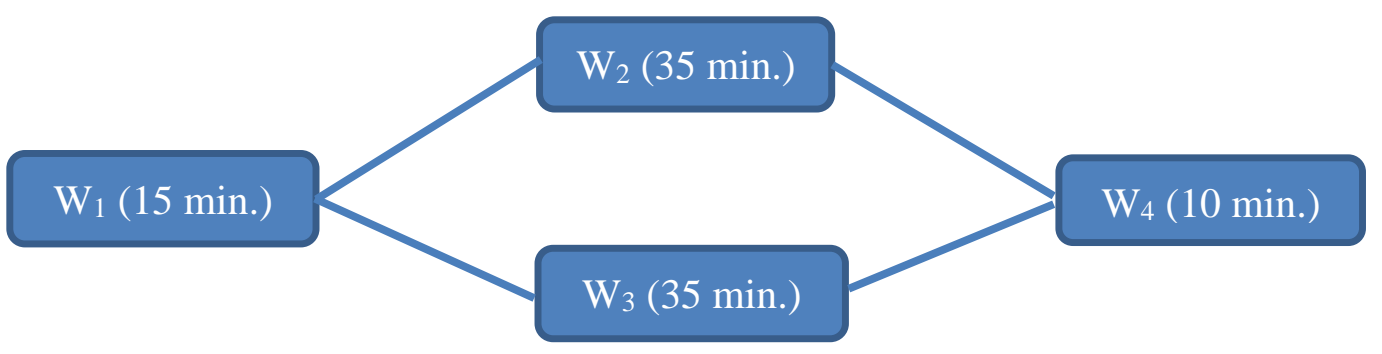

Fig. 2. Diagram 2

This means that after the same waiting, the $W_{4}$ worker would receive 2 semi-finished products for further processing and not one, and that the production bottleneck was partially overcome. From this simple example, it can be seen that interdisciplinarity and redistribution of workers can significantly affect the increase of production efficiency.

By applying the Lean concept of the Nagara system in one overhaul-production system, the number of workers decreased by $51.5 \%$, while the overhaul time increased by $3.1 \%$, which is satisfactory in this field of research. There is also a reduction in the entropy of the system that is created due to the presence of incompetent workers who refuse or prolong the execution of jobs due to constant requests for help in diagnosing the condition. More details on this use of human resources in the overhaul system can be found in [4], [5].

As we have seen, it is possible to significantly reduce the number of workers in a production plant without notable increase in time production. This is very important because in the conditions of a pandemic, production must be performed with a smaller number of workers in the plant or with reduced working hours. There are two options. The first option is to divide the number of workers of one production line into two sub-lines, each of which will work every other day. Another option is to work in four shifts of 6 hours each, while one quarter of the workers from each shift will make up that fourth line. In a pandemic, it is better to have more workers trained to perform more operations because production must not be allowed to stop due to the sudden illness of individuals. However, it should be noted that there is a fear among workers that they will be fired due to improved productivity. Reassurance must be given to workers that a higher level of individual training will lead to more paid workplaces and better distribution of workers to work tasks in terms of balancing norms. Worker needs to be reassured that he will not be laid off to improve productivity. Workers should be actively involved in improving performance, developing a climate of openness, respect and participation through KAIZEN meetings and thus creating mutual trust and respect between workers and management. Workers should be responsible for product quality. It is clear that the orientation of the organization of production towards worker is stronger. However, the management method cannot be completely simplified and reduced to the use of human resources, because the use of human resources is related to other components of Lean production and concept, as pointed out in the study [6]. In the end, even in the Lean concept, when it is necessary to reduce production costs, costs of worker training are reduced first. Thus, there is a real need to find a simple mathematical model, which will better allocate interdisciplinary workers with the aim of better productivity and which is easy to apply and modify in accordance with the existing situation. Such model will be presented in the next section.

\section{Mathematical model}

In mathematical modelling, we assume that all workers have accepted the Lean concept, which means awareness of the need for interdisciplinarity and redistribution to different work operations in order to increase work efficiency and reduce the number of workers on one production line. This concept assumes that workers are paid not only by experience but by ability to perform multiple different operations. Here we will identify work operations with the workplace and measure the complexity of the operation with a norm. More complex operations have a lower norm and less complex operations have a greater one. We will also assume that they want to redistribute workers without buying new machines. In the redistribution of workers, we will start from the redistribution of workers who do not already work on CNC machines, because great efficiency of production has already been achieved on these machines. There are production lines where a large number of operations cannot be performed on CNC machines. Such is, for example, the confection of the sewing line. We will denote workers as before with $W_{1}, W_{2}, \ldots, W_{n}$, work operations with $X_{1}, X_{2}, \ldots$, $X_{k}$ and the corresponding norm by $N_{1}, N_{2}, \ldots, N_{k}$. Each worker must be trained for at least two independent operations or for at least two work stations. We will assume that it is optimal for workers who do not work on CNC machines to be trained for three operations, provided that we can combine operations on the same machines or operations on the same type of machines. There are two strategies for redirecting human resources:

1. Redistribution was preceded by a serious analysis of the production process, the observation of production bottlenecks caused by the poor distribution of human resources and according to that, workers are trained for certain operations and after the training a new distribution is made.

2. Workers are already trained for certain operations and based on the available potential, redistribution is made. We have already stated that in the case of reducing production costs, even in the Lean concept, the training of workers should be abolished first. Therefore, it is necessary in the case we already have training funds that are less than the 
funds for the purchase of highly automated machines to approach the problem in a planned way and thus prefer the first strategy while the second is done in case of suspension of funds for training workers. By redistributing workers, we want to solve the following problem:

a) Solving bottlenecks in production caused by poor distribution of labour potential. But here we should be extremely careful because sometimes the problem is easily solved under the assumption that we have the option of introducing a new machine or purchase more expensive automated one, which is often not the case.

b) Balancing the norm or workload. It happens that some operations have a low norm due to their complexity, while others have a high norm due to the simplicity of the operation, which workers cannot achieve in real work time.

c) Increasing the efficiency and quality of production.

d) Reduction in the number of workers in the plant due to the COVID 19 pandemic.

We will analyse both strategies and offer appropriate mathematical models with the aim of solving problems a) - d). The mathematical model is required to be simple, adaptable and dynamic and thus easy to use. Since the set of workers is a discrete set from a mathematical point of view there is no need for excessive model complexity. Let us now consider the first strategy.

Suppose that all workers are already assigned to their jobs, and we want to train them further for the $l$ operations. Then we have

$$
\left(\begin{array}{c}
k-1 \\
l
\end{array}\right)=\frac{(k-1) !}{(k-1-l) ! l !}
$$

possibilities of choosing these additional operations. In our case

$$
\left(\begin{array}{c}
k-1 \\
2
\end{array}\right)=\frac{(k-1)(k-2)}{2}
$$

which is objectively a lot. Therefore, we will first locate the worker with the min norm $W_{\min }$ and the worker with the maximum norm $W_{\max }$ and mark their norms and work operations appropriately. Here we must be careful because $N_{\min }$ can refer to the minimal norm in the whole line or to one group of operations performed on the same type of machine. The same goes for the maximal norm. Please note that it is not desirable to send the same worker to several different machines. However, it is quite acceptable to train a worker who does manual work to work on at least one machine. An example of important manual work is quality control. A worker who works e.g. on the machine that performs the operation that is at the beginning of the production process, we can easily send to the product control in the last third of his working time. So the proposal is to perform a fusion of operations with the highest and lowest norm and add one of the adjacent operations. This is the reason for training workers for performing new tasks and operations. This way the problem of the production bottleneck is solved. We increase the input to the next operation not too much to overwhelm the worker, but again enough to speed up the production process. Therefore, we reduced the number of workers because we sent a worker away from the machine to one of the manual operations, in one part of the working hours, preferably at the beginning of production.

The new norm of workers in the designation $N^{\prime}$ where the bottleneck of production is calculated by formulae

$$
N_{j}^{\prime}=a \cdot N_{\max }+b \cdot N_{\min }+c \cdot N_{\text {adjacent }}, 0 \leq a \leq 1,0 \leq b \leq 1,0 \leq c \leq 1
$$

The exact value of $a, b$ and $c$ can be determined by a technologist or manager based on a recording of the production process or can be determined by applying fuzzy logic. More information about fuzzy logic can be obtained in [2]. In place of one worker at the bottleneck of production we introduce 2 workers $W_{\min }$ and $W_{\text {adjectant }}$ so that the worker $W_{\text {adjectant }}+1$ which usually has a higher norm gets instead of one semi-finished product two semi-finished products for processing. Usually $b=c$ is taken and $a$ is then easily calculated by $a=1-2 b$. It is possible to integrate similar operations which are too simple and are done on the same machine, meaning they have high norms, into one. This way we obtain new norm for the workplace

$$
N_{m}^{\prime}=a \cdot N_{m_{1}}+b \cdot N_{m_{2}}+c \cdot N_{m_{3}}, \quad 0 \leq a \leq 1,0 \leq b \leq 1,0 \leq c \leq 1 .
$$

Norm of the production line depends greatly from the first operation in line. To be exact, first operation defines it in some way because the number $A$ cannot be exceeded and is obtained as

$$
A=p_{1} \cdot N_{1}
$$

where $p_{1}$ is number of workers on the first operation. This is why it makes sense to combine work operations at the end of the production cycle with the first operation in the cycle, with the purpose of increasing the input of the semi products so we can get 


$$
N_{1}^{\prime}=a \cdot N_{1}+b \cdot N_{e_{1}}+c \cdot N_{e_{2}}, \quad 0 \leq a \leq 1,0 \leq b \leq 1,0 \leq c \leq 1
$$

Index $e$ denotes end operation. Since we can define wanted input we get

$$
A^{\prime}=p_{1}{ }^{\prime} \cdot N_{1}{ }^{\prime}
$$

where we define $A^{\prime}$ ourselves.

When calculating a new norm, we multiply the existing norm by the numbers $a, b$ and $c$. Since the norms must be natural numbers, we round the number of the norm to the first larger natural number.

\section{The Mathematical Model Application in Textile Line Production}

For our mathematical model application, we have chosen textile industry trousers production line. We observed process of trousers sewing because in this process CNC machine use is minimal. This means that in this process the interdisciplinarity of workers can be achieved and their redistribution to different work operations can bring significant results. The factory in which the production line is located has a fully automated tailoring department in which the located machines are Gerber Paragon LX Cutting System cutters, GERBER XLs50 Spreaders, GERBER MP+ Series Plotters, Aesus Labelers. For this paper we were more interested in sewing machines Veit automated Pants Finishers 8741, Veit Fusing Machines AX 450 C, Brother Industries NEXIO KE-430HS Electronic Direct Drive Lockstitch Bar Tackers, Brother Industries RH-9820 Eyelet Buttonhole, Brother Industries NEXIO T-8752D Twin Needle Direct Drive Split Needle Bar Lock Stitcher, Brother Industries NEXIO HE-800C Electronic Direct Drive Lockstitch Button Holer. In the production of trousers, the following operations are used, which take place according to the following schedule:

\begin{tabular}{|c|c|c|}
\hline Type of operation & $\begin{array}{c}\text { Norm for 1 } \\
\text { worker }\end{array}$ & $\begin{array}{c}\text { Number } \\
\text { of workers }\end{array}$ \\
\hline$X_{1}$ (processing) & 65 pieces & 2 \\
\hline$X_{2}$ (non-sewing operation) & 300 pieces & 1 \\
\hline$X_{3}$ (sewing operation) & 70 pieces & 2 \\
\hline$X_{4}$ (sewing operation) & 75 pieces & 2 \\
\hline$X_{5}$ (non-sewing operation) & 150 pieces & 1 \\
\hline$X_{6}$ (sewing operation) & 110 pieces & 1 \\
\hline$X_{7}$ (sewing operation) & 150 pieces & 1 \\
\hline$X_{8}$ (sewing operation) & 120 pieces & 1 \\
\hline$X_{9}$ (sewing operation) & 150 pieces & 1 \\
\hline$X_{10}$ (sewing operation) & 220 pieces & 1 \\
\hline$X_{11}$ (non-sewing operation) & 55 pieces & 2 \\
\hline$X_{12}$ (sewing operation) & 180 pieces & 1 \\
\hline$X_{13}$ (sewing operation) & 220 pieces & 1 \\
\hline$X_{14}$ (sewing operation) & 180 pieces & 1 \\
\hline$X_{15}$ (sewing operation) & 45 pieces & 3 \\
\hline$X_{16}$ (sewing operation) & 170 pieces & 1 \\
\hline$X_{17}$ (sewing operation) & 120 pieces & 1 \\
\hline$X_{18}$ (non-sewing operation) & 320 pieces & 1 \\
\hline$X_{19}$ (sewing operation) & 140 pieces & 1 \\
\hline$X_{20}$ (non-sewing operation) & 65 pieces & 2 \\
\hline$X_{21}$ (non-sewing operation) & 300 pieces & 1 \\
\hline$X_{22}$ (quality control operation) & 500 pieces & 1 \\
\hline$X_{23}$ (non-sewing operation) & 700 pieces & 1 \\
\hline
\end{tabular}

Table 1. Number of operations needed for trousers production and norm of workers

Total line production norm is 125 pieces done by 30 workers. Production bottlenecks occur in the production of welted pockets $\left(X_{3}, X_{4}\right)$, as well as in operations related to the placement of zippers $\left(X_{11}, X_{12}, X_{13}\right)$. The first bottleneck in production occurs when setting up welted pockets. We can overcome this by procuring pocket welting machines, however if we do not have enough funds to purchase a new machine, we can integrate operations, $X_{3}$ and $X_{4}$ with quality control $X_{22}$ as follows

$$
N_{3}{ }^{\prime}=N_{4}{ }^{\prime}=N_{22}{ }^{\prime}=0,5 \cdot N_{3}+0,4 \cdot N_{4}+0,1 \cdot N_{22}
$$

Workers perform product control in the last third of their working time. 
To solve problems related to the placement of the zippers we will integrate operations $X_{11}, X_{12} i X_{13}$. This way four workers will be sawing zippers instead of two. Their norms will be described as follows

$$
N_{11}{ }^{\prime}=N_{12}{ }^{\prime}=N_{13}{ }^{\prime}=0,6 \cdot N_{11}+0,2 \cdot N_{12}+0,2 \cdot N_{13}
$$

We can integrate many operations which are not related to sewing to further decrease number of workers. We can integrate the sewing operations if we need smaller number of workers on the production line because of the pandemic COVID 19.

\section{Conclusion}

Due to the problems of modern society such as the economic crisis and the COVID 19 pandemic, the distribution of human resources is once again coming into the focus of interest. On the other hand, production must be oriented towards the demands of consumers and the market. The way to address these conflicting requirements is to accept the Lean concept of thinking, which implies openness and trust between employees and management, as well as trust between employees themselves. The lean concept also implies the acceptance of additional training of workers to perform multiple work operations. It would be desirable for the training of workers to be planned, because with unplanned training, conditions of difficult production with delays can be created. An example of a problem that can occur in unplanned training of workers is reflected in the ability to train redundant workers for some work operations, while for other work operations there will be a shortage of manpower. It should also be considered that all funds for the planned training of workers should be used rationally. If production costs are reduced, the cost of training workers will be reduced first. A mathematical model is needed for the planned training of workers. Based on the mathematical model, a plan for training workers would be made, and later their redistribution. The goal is to provide efficient production, to enable production with a reduced number of workers in the event of a pandemic, whereby the worker would have the opportunity to make decisions in the production process.

Therefore, in this paper we have presented the mathematical model for redistribution of workers to different working places and operations. The presented mathematical model corresponds to the requirements of simplicity and dynamism and is not based on complex diagrams. We have seen that this way we can overcome bottled neck production and decrease the number of workers. For calculating coefficients $a, b$ and $c$ in our formulas one can use either the experience of technologist, engineers, workers and managers or apply fuzzy logic. At a specific plant, we showed how a quality result can be obtained by applying the proposed model. Further research will go in the direction of using the least squares method which is a mathematical method typical for solving engineering problems.

\section{References}

[1] Bozickovic, R. \& Maric, B. (2013). Lean Concept - a Challenge to Managers for the Better Future of the Company, Chapter 27 in DAAAM International Scientific Book 2013, Katalinic, B \& Tekic, Z. (Eds.), page numbers (491-510), DAAAM International, ISBN 978-3-901509-94-0, Viena, Austria

[2] Chen, G, \& Pham T. T. (2002). Introduction to Fuzzy Sets, Fuzzy Logic and Fuzzy Control Systems, CRC Press LLC, ISBN 0-8493-1658, New York

[3] Cvetkovic, N.; Moraca S.; Jovanovic, M.; Medojevic, M. \& Lalic, B. (2017). Enhancing the Agility and Performances of a Project with Lean Manufacturing Practices, Proceedings of the 28th DAAAM International Symposium, 08-11th November 2017, ISBN 978-3-902734-11-2, Katalinic, B. (Ed.), pp.0661-0670, DAAAM International Viena, Austria, DOI 10.2507/28 th.daaam.proceedings093

[4] Maric, B.; Bozickovic, R. \& Cosic, I. (2010). Nagara System for Planning Human Potentials in the Repair Process of Technical Systems, Process of Technical Systems, Proceedings of Conference Maintenance 2010, 10-13 June 2010, ISSN 1986-583x, Brdarevic, S.\& Jasarevic, S. (Eds.) pp. 23-28, University of Zenica, Faculty of Mechanical Engineering in Zenica, Zenica Bosnia and Herzegovina

[5] Maric, B.; \& Bozickovic, R. (2014). Maintenance of Technical Systems \& Lean concept, Faculty of Mechanical Engineering, East Sarajevo, University of East Sarajevo, ISBN 978-99976-623-0-9, East Sarajevo

[6] Olegehe, O. \& Salonitis, K. Hybrid simulation modelling of the human-production process interface in lean manufacturing systems. International Journal of Lean Six Sigma, Vol., 10, No., 2, (2019)., page numbers (665690), IJLSS-01-208-0004

[7] Softic, A.; Basic, H.; Lulic, H. \& Nakic, A. (2020). Application of the Lean Concept for Analysis and Optimization of the Automobile Filter Production Company, Proceedings of the 31th DAAAM International Symposium, 21-24th October 2020, ISBN 978-3-902734-29-7, Katalinic, B. (Ed.), pp.0708-0716, DAAAM International Viena, Austria, DOI 10.2507/31st.daaam.proceedings098

[8] Vujica Herzog, N. \& Buchmeister, B. (2012). Lean Manufacturing in Slovenian Companies, Chapter 11 in DAAAM International Scientific Book 2012, Katalinic, B. (Ed.), page numbers (117-128), DAAAM International, ISBN 978-3-901509-86-5, Viena, Austria 